A

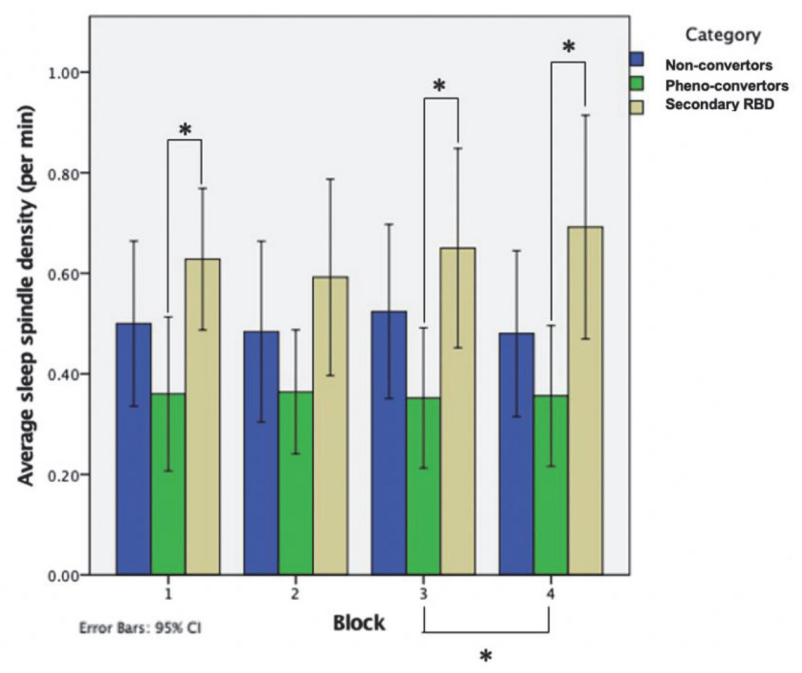

B

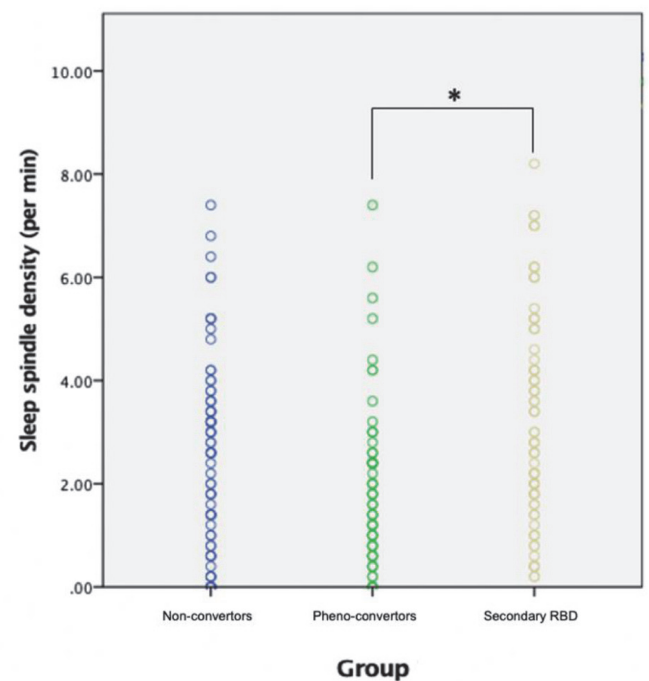

Abstract 54 Figure 1 Sleep spindle density. A) The average spindle density per minute in each block of NREM throughout the night ${ }^{\prime * \prime}=\mathrm{p}<0.05$ (One-way ANOVA and post-hoc Tukey's test)

Abbreviations: SS/min=Sleep spindles per minutes. Error bars show a 95\% confidence interval

known to decrease in patients with alpha-synucleinopathies. We hypothesized that that sleep spindle density would differ in patients with iRBD with and without an alphasynucleinopathy.

Methods This was a retrospective cohort study comprised of sixty male participants, all of whom were diagnosed with RBD. Sleep spindles were manually identified by an experienced technologist at a central scalp location (C3-A2) in 20 patients with $\mathrm{RBBD}$ who converted to an alpha-synucleinopathy, 20 age-matched patients with secondary RBD and 20 patients with a diagnosis of iRBD who had not converted.

Results Patients who phenoconverted showed a significant decrease in sleep spindle activity compared to patients with secondary RBD $(\mathrm{p}<0.05)$ at time of diagnosis. Spindle density was lower in patients who had phenoconverted. Sleep spindle density reduction was significant in patients with $\mathrm{PD}$; there were too few patients with MSA and DLB to determine differences in spindle density counts.

Discussion This is the first study to look into multiple alpha-synucleinopathies to investigate sleep spindle density changes. As a statistical significance was found between the spindle density of patients who had gone on to develop a neurodegenerative disorder and those who had secondary RBD, when examining spindle density in the second half of the night $(p=0.026)$ (figure 1$)$. It follows that sleep spindle density may have the potential to be a prodromal marker for phenoconversion and underlying alterations in sleep networks that lead to a clinical diagnosis of an alphasynucleinopathy.

\section{THE USE OF TELEMEDICINE IN THE MANAGEMENT OF CONTINUOUS POSITIVE AIRWAY PRESSURE FOR THE TREATMENT OF OBSTRUCTIVE SLEEP APNOEA, A RANDOMISED CONTROLLED TRIAL}

${ }^{1}$ Tracy Jones*, ${ }^{2}$ Rebecca Stores, ${ }^{2}$ Jenny Roddis. ${ }^{1}$ sle of Wight Nhs Trust, Isle of Wight, UK; ${ }^{2}$ University of Portsmouth, Portsmouth, UK

\subsection{6/bmjresp-2021-bssconf.49}

Introduction Obstructive sleep apnoea (OSA) is a condition whereby the airway partially or totally obstructs during sleep. The gold-standard treatment for moderate to severe OSA is continuous positive airway pressure (CPAP). However compliance with treatment can be troublesome with $20-30 \%$ of patients stopping treatment. A recent meta-analytic review (Aardoom et al 2000) of telemedicine in CPAP treatment has

Abstract 55 Table 1 Compliance in hours for each study arm

\begin{tabular}{|c|c|c|c|c|}
\hline $\begin{array}{l}\text { Data } \\
\text { Collection } \\
\text { Points }\end{array}$ & $\begin{array}{c}\text { Compliance } \\
\text { (hours in } 24 \text { hours } \\
\text { use) }\end{array}$ & $\begin{array}{c}\text { Arm } 1 \text { - Standard } \\
\text { Care, face to face } \\
\text { follow up } \\
\text { appointment } \\
\quad(n=30)\end{array}$ & $\begin{array}{c}\text { Arm } 2 \text { - Modem } \\
\text { and virtual follow } \\
\text { up clinic } \\
\text { appointment } \\
\quad(n=28)\end{array}$ & $\begin{array}{l}\text { Arm } 3 \text { - Modem, } \\
\text { application } \\
\text { DreamMapper and } \\
\text { virtual follow up } \\
\text { appointment } \\
(n=32)\end{array}$ \\
\hline \multirow{2}{*}{$\begin{array}{l}1^{\text {st }} \text { follow up 1- } \\
2 \text { weeks }\end{array}$} & Mean & 6.18 & 5.65 & 6.54 \\
\hline & SD & 2.21 & 2.40 & 1.89 \\
\hline \multirow{2}{*}{$\begin{array}{l}6 \text { month follow } \\
\text { up }\end{array}$} & Mean & 6.28 & 5.49 & 6.24 \\
\hline & SD & 1.54 & 2.17 & 1.65 \\
\hline
\end{tabular}


concluded that the effectiveness of telemedicine on adherence remains undecided.

Methods A non-blinded, single centre, randomised controlled trial was carried out with patients randomised to 1 of 3 arms (arm 1 standard care and face-to-face appointments; arm 2 modem and virtual follow up clinic; arm 3 modem, Dreammapper $^{\mathrm{TM}}$ and virtual follow up appointment). Consecutive moderate-severe OSA patients requiring CPAP were recruited to the study to a sample size of 90. Data was collected at first appointment, at 1st follow up from commencing CPAP (1-2 weeks) and at 6 months from commencing CPAP. Data collection included patient's demographics (table 1), CPAP compliance (average hours of usage in 24 hours), mask leak, apnoea/hypopnoea index (AHI) and Epworth sleepiness scores (ESS). Qualitative data is currently being collected via interviews to explore the patient experience in each arm of the study.

Discussion Preliminary analysis (table 2) indicates that arm 3 demonstrated significant better compliance of 36 minutes at first follow up compared to arm 1, with compliance at 6 months between both these arms levelling out with no significant difference at 6 months. The patient group in arm 2 demonstrated a significantly reduced in compliance compared to the other two groups at both week 1-2 and 6 months. Preliminary results suggest that the type of telemedicine intervention could impact the early compliance experienced by patients.

\section{REFERENCE}

1. Aardoom JJ, Loheide-Niesmann, Ossebaard HC, Riper H. Effectiveness of eHealth Interventation in improving treatment adherence for adults with obstructive sleep apnoea: meta-analytic review. Journal of Medical Internet Research 2020 Feb;22 (2): 1-12

\section{ROLE OF SLEEP DURATION IN CARDIOVASCULAR MORTALITY AMONG PATIENTS WITH CHRONIC KIDNEY DISEASE}

Sri Banerjee*, Ubong Usua. Walden University, Leola, USA

10.1136/bmjresp-2021-bssconf.50

Growing evidence suggests an association between both short and long duration of habitual sleep and adverse health outcomes. In order to determine whether the population longitudinal evidence supports the presence of a relationship between Chronic Kidney Disease (CKD) and cardiovascular disease (CVD)-mortality, and how sleep duration plays a role in mortality outcomes.

This is a population-based cohort study of National Health and Nutrition Examination Surveys participants between 2005 and 2010 with mortality data obtained through 2015. Adults aged 20 years or older with sleep duration information were categorized into excessive ( $\geq 8$ hours) versus normal. CKD was determined using the Cockcroft-Gault equation and considered positive at $\mathrm{GFR}<60 \mathrm{~mL} / \mathrm{min}$. Outcomes of CVD-mortality were evaluated using Cox regression.

The percentage of deaths from low sleep duration among the population $(\mathrm{N}=15,586)$ were higher among individuals without High School (HS) Diploma (12.8\%) versus at least some college education (5.7\%). The mean follow-up was 7.7 years. For CVD-mortality, the overall unadjusted hazard ratio (HR) of individuals with CKD to no CKD was $1.54(95 \%$ confidence interval $[\mathrm{CI}], 1.11-2.14, \mathrm{p}=0.01)$. Adjusted HR was elevated, 1.59 (CI 1.01-2.52, p = .04), among those with excessive sleep duration but closer to 1.0 (0.62 CI $0.25-1.51$, $\mathrm{p}<0.25)$ among individuals with normal sleep duration, after controlling for medical (obesity, diabetes, and C-reactive protein) and demographic risk factors (age, gender, povertyincome-ratio, education, and ethnicity).

Our study shows an unambiguous and consistent pattern of increased risk of dying from cardiovascular disease among individuals with CKD. However, this relationship is especially pronounced among individuals who have excessive sleep duration, making it an important determinant of health. Screening for sleep disorders is especially important in the consideration of other chronic diseases like cardiorenal syndrome.

\section{UK ADHERENCE RATES TO CONTINUOUS POSITIVE AIRWAY PRESSURE BEFORE AND AFTER THE START OF THE CORONAVIRUS PANDEMIC}

${ }^{1}$ Julia Dielesen*, ${ }^{2}$ Peter Dickel, ${ }^{3}$ David R Jones, ${ }^{3}$ A Siddiq Pulakal, ${ }^{4}$ Neil Ward, ${ }^{5}$ Justin C Pepperill, ${ }^{6}$ Simon Merritt, ${ }^{7,8}$ Joerg Steier, ${ }^{1,2,8} \mathrm{~A}$ Sathyapala. ${ }^{1}$ National Heart and Lung Institute, Imperial College London, London, UK; ${ }^{2}$ Harefield Hospital, Guy's and St Thomas's NHS Foundation Trust, Middlesex, UK; ${ }^{3}$ Wythenshawe Hospital, Manchester, UK; ${ }^{4}$ Derriford Hospital, Plymouth, UK; ${ }^{5}$ Musgrove Park Hospital, Taunton, UK; ${ }^{6}$ Conquest Hospital, Hastings, UK; ${ }^{7}$ St. Thomas's Hospital, Harefield Hospital, UK; ${ }^{8}$ King's Health Partners, London, UK

\subsection{6/bmjresp-2021-bssconf.51}

Introduction The most efficacious treatment for obstructive sleep apnoea (OSA) is Continuous Positive Airway Pressure (CPAP). CPAP's benefit is curtailed however, because many patients do not adhere to treatment, estimated from trials at 17-85\%. ${ }^{1}$ Since the Covid-19 pandemic, US and French CPAP adherence rates have been reported to have changed. ${ }^{2}$ We sought to determine CPAP adherence rates of NHS patients pre-and post-pandemic.

Methods 5 sleep centres were recruited. To detect an 18\% difference [US Philips Respironics survey\% adherent 36\% (2019) vs $18 \%$ (2020)], $80 \%$ power, p $0.05 \%$, 2-tailed test, $\mathrm{n}=92$. Average use at Night 3, 7, 14, 1 and 3 months and clinical data collected from 100 patients starting CPAP for first time in i) April 2019 and ii) September 2020, using Resmed Airview and clinical records. Adherence defined as CPAP use $\geq 4$ hrs/night for $\geq 70 \%$ of nights, or at Night 3 median use $\geq 4$ hrs.

Results See table 1 for patient characteristics. In 2019, in all centres, $\%$ of adherent patients fell from Night 3 to 3 months, with 3-month adherence rates between 29\%-50\% $(p=0.025$, figure 1). Similar was seen in 2020 in Conquest $(\mathrm{CH})$ and Musgrove Park hospitals (MSH); in $\mathrm{CH}$, higher\% seen up to 1 month compared to 2019. In 2020, at Manchester (MH), Derriford (DH) and Guys and St Thomas's hospitals (GSTT) the $\%$ of adherent patients did not fall between Night 3 and 3 months; $\mathrm{MH}$ and $\mathrm{DH}$ maintained similar\% to 2019, in GSTT, there was a smaller\% starting at Night 3 compared to 2019 (30\% vs 55\%, p<0.0001). 2020 3 -month adherence rates were different between centres (27$51 \%, \mathrm{p}=0.004)$.

Discussion 3 month adherence rates are low -at best 50\%. Rates have changed at some centres since the pandemic, with change varying depending on the treatment pathway modifications implemented. 